\title{
Effect of harmonic magnetic field and pulse magnetic field on microstructure of high purity Cu during electromagnetic direct chill casting
}

\author{
Lei Bao ${ }^{1,2}$, *Da-zhi Zhao ${ }^{2}$, Yin-ji Zhao", ${ }^{1,}$ Yong-hui Jia, ${ }^{1,2}$ Xuan Wang', ${ }^{1,}$ Qi-chi Le ${ }^{1,2}$ \\ 1. Key Lab of Electromagnetic Processing of Materials, Ministry of Education, Northeastern University, Shenyang 110819, China \\ 2. School of Materials Science and Engineering, Northeastern University, Shenyang 110819, China
}

\begin{abstract}
The effects of two types of magnetic fields, namely harmonic magnetic field (HMF) and pulse magnetic field (PMF) on magnetic flux density, Lorentz force, temperature field, and microstructure of high purity Cu were studied by numerical simulation and experiment during electromagnetic direct chill casting. The magnetic field is induced by a magnetic generation system including an electromagnetic control system and a cylindrical crystallizer of $300 \mathrm{~mm}$ in diameter equipped with excitation coils. A comprehensive mathematical model for high purity $\mathrm{Cu}$ electromagnetic casting was established in finite element method. The distributions of magnetic flux density and Lorentz force generated by the two magnetic fields were acquired by simulation and experimental measurement. The microstructure of billets produced by HMF and PMF casting was compared. Results show that the magnetic flux density and penetrability of PMF are significantly higher than those of HMF, due to its faster variation in transient current and higher peak value of magnetic flux density. In addition, PMF drives a stronger Lorentz force and deeper penetration depth than HMF does, because HMF creates higher eddy current and reverse electromagnetic field which weakens the original electromagnetic field. The microstructure of a billet by HMF is composed of columnar structure regions and central fine grain regions. By contrast, the billet by PMF has a uniform microstructure which is characterized by ultra-refined and uniform grains because PMF drives a strong dual convection, which increases the uniformity of the temperature field, enhances the impact of the liquid flow on the edge of the liquid pool and reduces the curvature radius of liquid pool. Eventually, PMF shows a good prospect for industrialization.
\end{abstract}

Key words: high purity Cu; pulse magnetic field; harmonic magnetic field; microstructure; sputtering target; direct chill casting

CLC numbers: TG146.1 1 Document code: A Article ID: 1672-6421 2021 02-141-06

\section{Introduction}

With the rapid development of very large-scale integration (VLSI) and flat panel display (FPD), the demands for sputtering target materials with high purity are greatly increased ${ }^{[1]}$. High purity $\mathrm{Cu}$ is one of the most commonly used metals for sputtering target ${ }^{[2]}$.

Generally, the sputtering rate, deposited film quality and thickness uniformity are high when using a target with fine grains and small grain size difference ${ }^{[3]}$. Therefore,

\section{*Da-zhi Zhao}

Male, Ph. D., Lecturer. His research interests are mainly focused on solidification process, casting technology, especially direct chill casting, and nonferrous metals (aluminum and copper alloys). He has published over twenty papers in international and domestic journals, and holds dozens of invention patents of China.

E-mail: zhaodz@smm.neu.edu.cn;

Received: 2020-08-14; Accepted: 2020-11-11 whether a high-performance high purity $\mathrm{Cu}$ target can be obtained depends on whether fine and uniform grains can be obtained during the production process.

As-cast microstructure of a high purity $\mathrm{Cu}$ billet, especially a large-scale billet, produced by direct chill (DC) casting is normally characterized by highly developed coarse columnar grains that result in poor sputtering performance properties when used as a sputtering target ${ }^{[1]}$. To refine grains and improve microstructure, electromagnetic vibration has been successfully introduced in industrial DC casting for large-scale steel, $\mathrm{Al}$ and $\mathrm{Mg}$ alloy billets because of its advantages of remarkable grain refinement and low $\operatorname{cost}^{[4,5]}$. The logical conclusion is that electromagnetic vibration has a high likelihood of being effective on grain refinement for a DC billet of high purity $\mathrm{Cu}$.

In the practical application of electromagnetic vibration, the harmonic magnetic field (HMF) and pulse magnetic 
field (PMF) are widely used ${ }^{[6]}$. HMF is generated by continuous alternating current and characterized by real-time changing magnetic force and magnetic direction following a sine wave. Similarly, PMF is generated by transient pulse current, and the magnetic field changes periodically. Different types of electromagnetic fields change the solidification behavior during casting in different ways, and finally result in various as-cast microstructures ${ }^{[7]}$. For example, HMF with low frequency was reported to be effective in grain refinement, as well as microstructure and surface quality improvement for DC cast ferrous and nonferrous metals ${ }^{[8]}$. In very recent years, PMF also shows a demonstrable effect in grain refinement and microstructure improvement of $\mathrm{Mg}$ alloy by $\mathrm{DC}$ casting, and it is considered a novel promising method for microstructure improvement of metallic materials ${ }^{[9-11]}$. However, studies on the mechanism of how PMF alters the solidification behavior of metals during casting have not yet been investigated as extensively as for HMF.

Therefore, this work aims to reveal the effects of HMF and PMF on grain refinement for high purity $\mathrm{Cu}$ billet by investigating the magnetic flux density, Lorentz force and temperature field in the industrial casting system and production process. The magnetic flux density was measured by Tesla meter and the microstructure improvement was evaluated based on the comparison of the grain size and homogeneity. A comprehensive mathematical model of high purity $\mathrm{Cu}$ electromagnetic casting was established using the finite element method. Finally, the mechanism of action of the two magnetic fields on high purity $\mathrm{Cu}$ melt was discussed.

\section{Experimental details and mathematical model}

\subsection{Materials and magnetic generation system}

The composition of the high purity $\mathrm{Cu}$ used in this work is listed in Table 1.

Table 1: Composition of high purity $\mathrm{Cu}$ (wt.\%)

$\begin{array}{cccccccc}\mathrm{Cu} & \mathrm{Si} & \mathrm{S} & \mathrm{P} & \mathrm{Fe} & \mathrm{Al} & \mathrm{O} & \text { Impurity } \\ >99.993 & 0.0005 & 0.001 & 0.002 & 0.001 & 0.001 & 0.001 & <0.001\end{array}$

Figure 1 schematically shows the magnetic field generation system adopted in this work, including an electromagnetic control system and a crystallizer equipped with a set of excitation coils. Magnetic field is generated by an electromagnetic control system that is capable of generating sine wave current and pulse current. The crystallizer with a diameter of $300 \mathrm{~mm}$ is composed of an inner sleeve, an outer sleeve, a water circulating system and a set of excitation coils.

The electromagnetic control system is used to induce a magnetic field and an eddy current simultaneously inside the crystallizer during electromagnetic casting, and their interaction with each other generates Lorentz force acting on the high purity $\mathrm{Cu}$ melt ${ }^{[12]}$. Then, the Lorentz force agitates the $\mathrm{Cu}$ melt

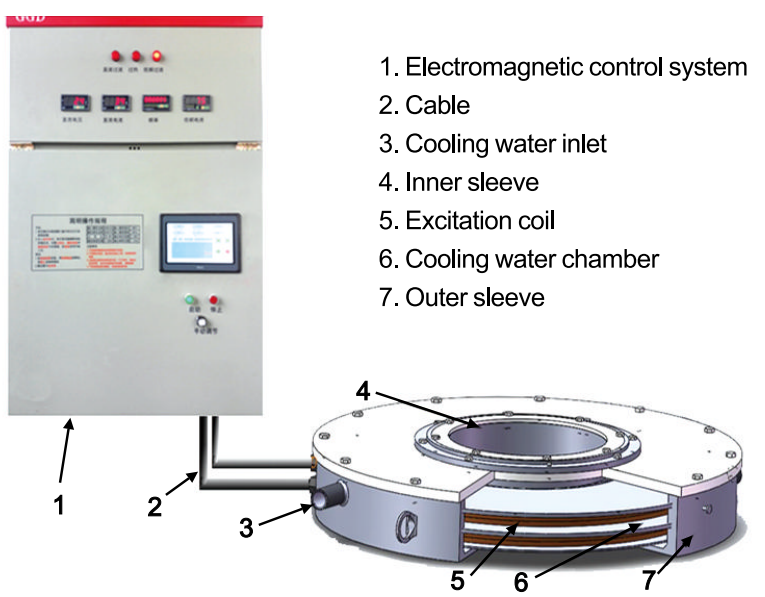

Fig. 1: Schematic diagram of magnetic field generation system

and enhances the transfer of momentum and energy during solidification by forced convection ${ }^{[13,14]}$. The working principle can be summarized as follows: AC voltage is transformed into unidirectional pulsating DC voltage by thyristor module composed of rectifying elements. Damping oscillation current is generated by IGBT controller and electrodeless capacitor through capacitor bank filtering. Signal output is controlled by PLC core component, and then the corresponding magnetic field is generated inside the crystallizer through the excitation coil. HMF is generated by sine wave current flowing through the excitation coil and PFM is generated by pulse current generated by pulse power system that is a part of the electromagnetic control system. Figure 2 shows the waveforms of sine wave current and pulse current during a few cycles measured by a current oscilloscope.

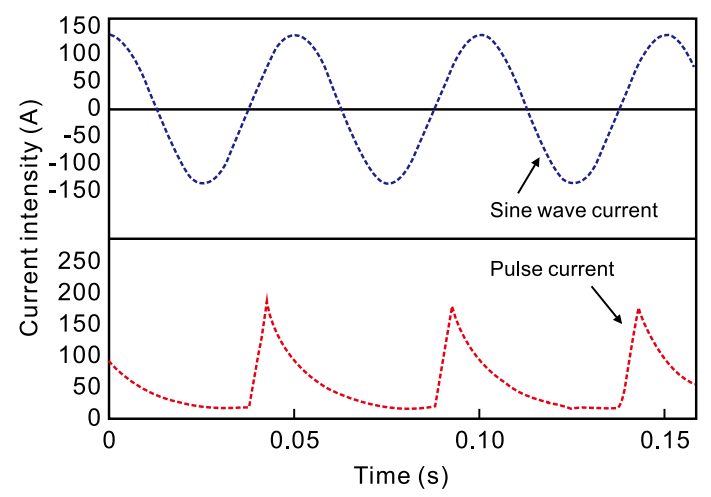

Fig. 2: Measured waveforms of sine wave current and pulse current

\subsection{Experimental methods}

The high purity $\mathrm{Cu}$ was firstly melted in an induction furnace at $1,220^{\circ} \mathrm{C}$. Then, the melt was held for $20-30 \mathrm{~min}$ at $1,180^{\circ} \mathrm{C}$ before it was poured into the crystallizer to produce a cylindrical billet with a diameter of $300 \mathrm{~mm}$ and a length of $4 \mathrm{~m}$. The magnetic field generation system was switched on $10 \mathrm{~min}$ before the start of casting. The casting speed was $4.8 \mathrm{~m} \cdot \mathrm{h}^{-1}$ and the velocity of the cooling water flow was $15 \mathrm{~m}^{3} \cdot \mathrm{h}^{-1}$. The parameters of HMF were set as follows: 140 A current intensity, $20 \mathrm{~Hz}$ 
frequency, and 80-turn coil. The parameters of PMF were $80 \mathrm{~A}$ current intensity, $20 \mathrm{~Hz}$ frequency, $20 \%$ duty cycle, and 80 turn coil. During casting, a Tesla meter sensor protected by a corundum tube was immersed into the melt to measure the magnetic flux density. A high temperature thermocouple was also placed into the melt for collecting the temperature data. Finally, the samples for microstructure observation were taken from the center of the billet and polished by a traditional approach for metallographic preparation, and then etched with a solution of $30 \%$ nitric acid. The average grain size was measured by the intercept method.

\subsection{Numerical modeling}

In this work, the discretized equations for electromagnetic field were calculated using the finite element method. In order to accurately describe the interaction of multiple physical fields in the solidification process of high purity $\mathrm{Cu}$ under the action of magnetic field, the coupling calculation on electromagnetic field, melt flow, heat transfer and melt solidification is needed ${ }^{[15]}$. This calculation uses a model based on Maxwell's equations composed of Ampere's loop law, Faraday's law of electromagnetic induction, Gauss' law of electric flux and Gauss' law of magnetic flux. A single region volume average model was used to simulate the distribution of temperature field and flow field in the casting. The model integrates all the model equations through a governing equation in the whole solidification process, and defines each region by calculating the energy distribution. In this model, the displacement current was ignored due to the excellent conductivity of high purity $\mathrm{Cu}$ melt. The change of flow field was assumed to have no influence on the distribution of electromagnetic field owing to the Reynolds number being far less than $1^{[16]}$. The material properties for calculation and simulation are listed in Table 2.

Table 2: Material properties for magnetic field simulation

\begin{tabular}{cccc} 
Component & Materials & Relative permeability & Resistivity $(\Omega \cdot \mathbf{m})$ \\
\hline Metal melt & Pure Cu (liquid) & 1 & $8.2 \times 10^{-8}$ \\
Inner sleeve of crystallizer & Cu alloy & 1 & $7.1 \times 10^{-8}$ \\
Excitation coil & Pure Cu (solid) & 1 & $1.7 \times 10^{-8}$ \\
Air zone & Air & 1 & $\infty$
\end{tabular}

Figure 3 shows the fitted current waveforms of HMF and PMF used in calculation. The current of HMF (blue curve in Fig. 3) reaches its positive and negative maximum at $0.0125 \mathrm{~s}$ and $0.0375 \mathrm{~s}$, respectively, and the current of PMF (red curve in Fig. 3) reaches its peak value at $0.0090 \mathrm{~s}$.

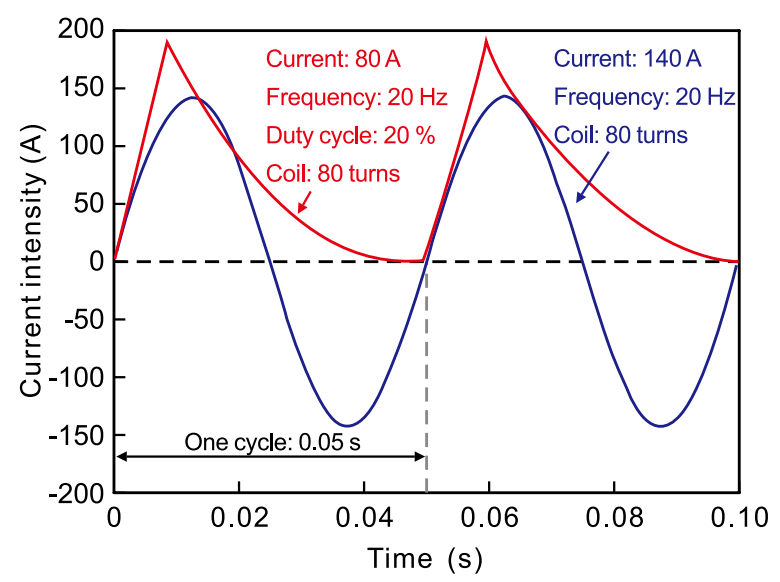

Fig. 3: Fitted current waveforms of HMF and PMF used in calculation

\section{Results and discussion}

\subsection{Distribution of magnetic flux density}

Figure 4 shows the magnetic flux density inside the crystallizer filled with high purity $\mathrm{Cu}$ melt under HMF and PMF at $0.0090 \mathrm{~s}$, $0.0125 \mathrm{~s}, 0.0250 \mathrm{~s}$ and $0.0375 \mathrm{~s}$ in one cycle $(0.05 \mathrm{~s})$. The reason for choosing above instant for analysis is that, from the current waveforms in Fig. 3, it is clear that the maximum values of magnetic flux density of HMF and PMF present at $0.0125 \mathrm{~s}$, $0.0375 \mathrm{~s}$ and $0.0090 \mathrm{~s}$, and the instant of half-cycle, $0.0250 \mathrm{~s}$, are also selected. It can be seen from Fig. 4 that under the effect of HMF and PMF, the maximum values of magnetic flux density are $88 \mathrm{mT}$ and $117 \mathrm{mT}$, respectively. Note that due to the skin effect of alternative electromagnetic field, the maximal magnetic flux density is always located in the area close to the surface of crystallizer, therefore, the penetration depth of the electromagnetic field through the inner sleeve of the crystallizer is limited ${ }^{[17]}$. This can be proved by the horizontal distribution of maximum magnetic flux density from center to surface of crystallizer under the two magnetic fields shown in Fig. 5. It can be found that because of magnetic field attenuation and shielding effect, the magnetic flux density near the crystallizer surface is much higher than that near the crystallizer center. Moreover, the attenuation rate of magnetic flux density under PMF is much lower than that under HMF.

The range of maximum magnetic flux density of PMF distributes nearly all over the cross-section of crystallizer (indicated by blue dash in Fig. 4), indicating the excellent penetrability of PMF. The reason why PMF has higher maximal magnetic flux density and the penetration depth than those of HMF is that the variation in transient current for PMF is much faster than that of HMF, and moreover, PMF has a higher peak value of magnetic flux density. 

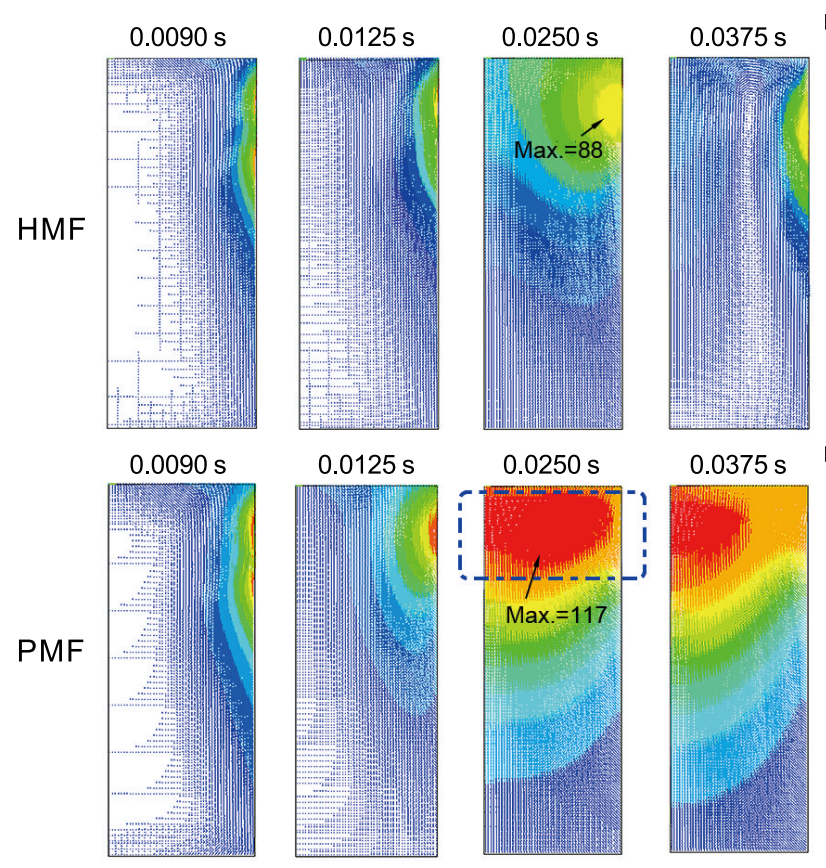

Magnetic flux
density $(\mathrm{mT})$

$0.0125 s$
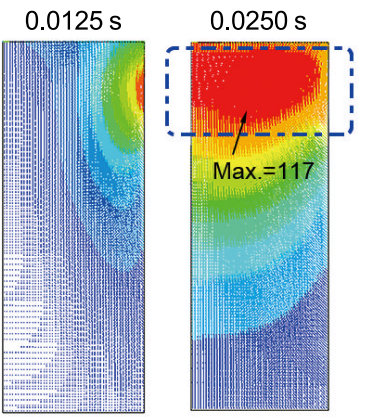

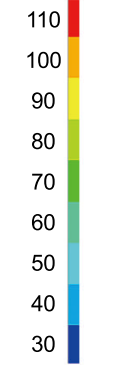

$0.0375 \mathrm{~s} \quad \begin{gathered}\text { Magnetic flux } \\ \text { density (mT) }\end{gathered}$

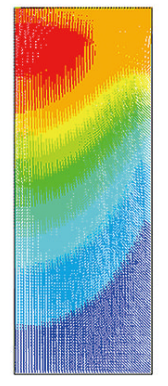

Fig. 4: Magnetic flux density inside crystallizer under HMF and PMF at 0.0090 s, $0.0125 \mathrm{~s}, 0.0250 \mathrm{~s}$ and $0.0375 \mathrm{~s}$

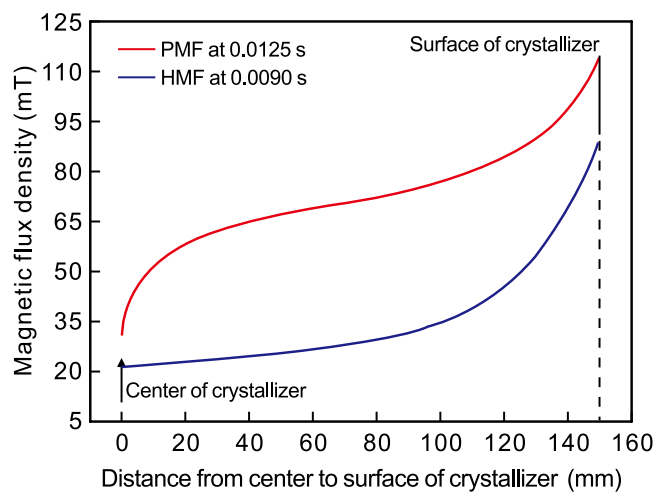

Fig. 5: Horizontal distribution of magnetic flux density from center to surface of crystallizer under HMF and PMF

\subsection{Distribution of Lorentz force}

Figure 6 shows the distribution of Lorentz force acting on the melt under HMF and PMF at $0.0090 \mathrm{~s}, 0.0125 \mathrm{~s}, 0.0250 \mathrm{~s}$ and $0.0375 \mathrm{~s}$, respectively. It can be found that the maximum Lorentz force by PMF is $0.103 \mathrm{~N}$, which is much higher than that by HMF $(0.087 \mathrm{~N})$. Moreover, under PMF, the horizontal and vertical penetration depth of Lorentz force are all higher than those of HMF, because HMF creates a higher eddy current in the sleeve and $\mathrm{Cu}$ melt compared with PMF. This eddy current creates a reverse electromagnetic field which weakens the original electromagnetic field generated by source current flowing in coil, and eventually results in a lower Lorentz force and penetration depth ${ }^{[18]}$.

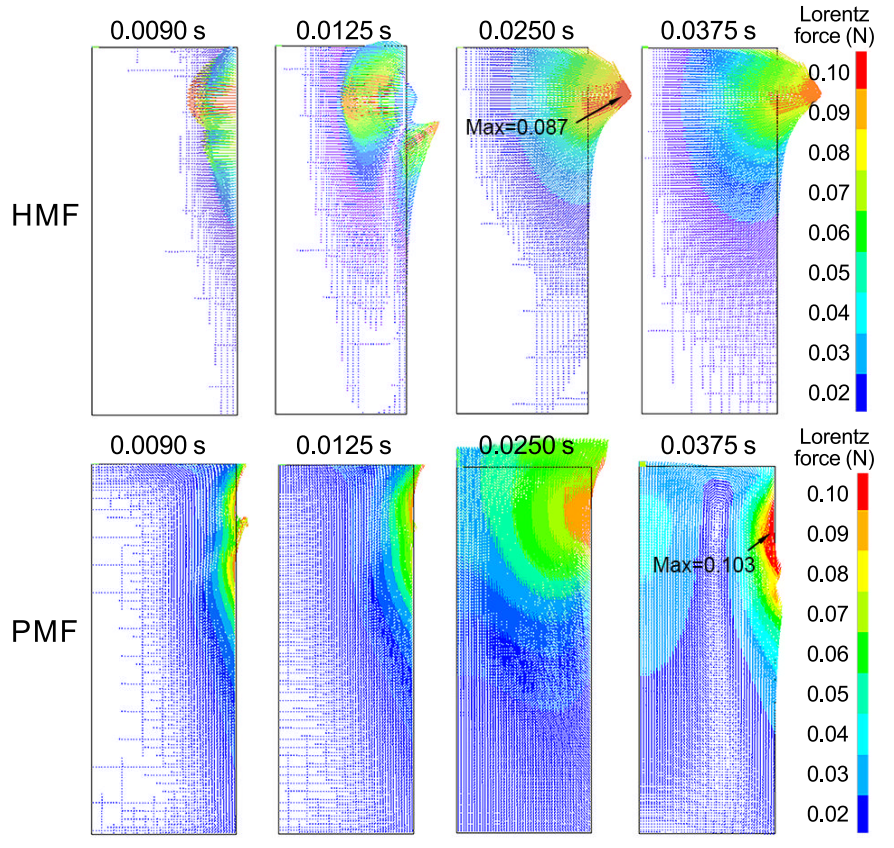

Fig. 6: Distribution of Lorentz force inside crystallizer under HMF and PMF at 0.0090 s, $0.0125 \mathrm{~s}, 0.0250 \mathrm{~s}$ and $0.0375 \mathrm{~s}$ 


\subsection{Temperature and flow fields of high purity Cu melt}

Figure 7 shows the temperature and flow fields of high purity $\mathrm{Cu}$ melt at stable stage in HMF and PMF casting. It can be found that, compared with $\mathrm{HMF}$, the isotherm from crystallizer surface to center under PMF is more steady and smooth as indicated by red dash lines in Fig. 7, which means the temperature field under PMF is more uniform than that under HMF. Regarding the flow field of the melt, it can been seen that high temperature melt is pushed to the crystallizer surface and low temperature melt flows reversely under forced convection caused by the magnetic field. Both HMF and PMF drive strong convection. A pair of dual convection flows driven by the Lorentz force of PMF are observed. This dual convection contributes to the steady and smooth isotherm. Therefore, PMF is effective on temperature field homogenization, which is the key factor affecting the solidification behavior and microstructure of the billet.

\section{HMF}

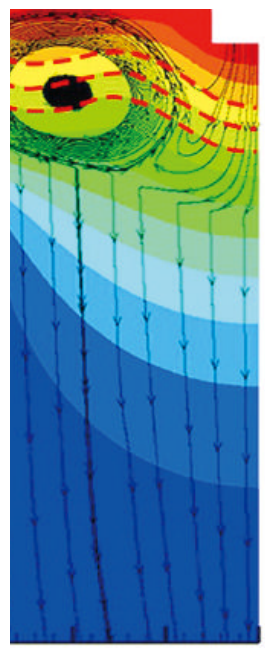

PMF

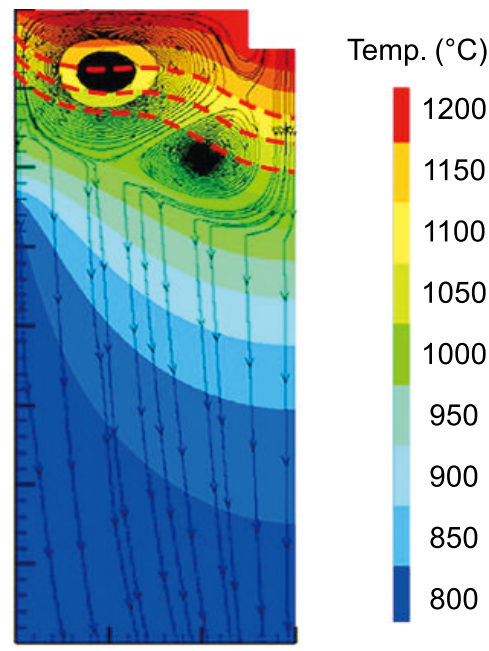

Fig. 7: Temperature and flow fields of high purity $\mathrm{Cu}$ melt in HMF and PMF casting

\subsection{Microstructure of high purity Cu billets by HMF and PMF casting}

Figure 8 shows the microstructure on the longitudinal section taken from the central section of billets produced by HMF and PMF casting. The microstructure of the billet by HMF casting is composed of a columnar structure region [red dash line area in Fig. 8(a)], and a central fine grain region [green dash line area in Fig. 8(a)]. In the columnar structure region, highly developed columnar grains with the average size of 2,576 $\mu \mathrm{m}$ can be seen and these columnar grains grow along the temperature gradient direction [blue dash line in Fig. 8(a)]. The grains in the central fine grain region are much smaller (the average size is $415 \mu \mathrm{m}$ ) and are elongated in temperature gradient direction as well. The billet by PMF casting has a uniform microstructure characterized by ultra-fine and uniform grains with the average size of $187 \mu \mathrm{m}$. These refined columnar grains also grow along the temperature gradient direction, as shown in Fig. 8(b).

When an electromagnetic field is applied on the $\mathrm{Cu}$ melt, forced convection is induced by Lorentz force in the liquid zone and mushy zone, which results in relative movement between solid and liquid in the mushy zone due to the different resistivity of solid and liquid ${ }^{[12]}$. This relative movement is able to promote the heat transfer and make temperature distribution uniform. During solidification, the liquid pool becomes shallow and the angle between columnar grain growth direction and casting direction decreases, thus the solidification structure presents smaller grain size ${ }^{[15,16]}$.

According to Ref. [18], the common mechanism of the microstructure improvement by magnetic field can be attributed to the uniform temperature distribution, shallow liquid pool and strong convection that are all not favorable for the growth of coarse columnar grain. In this work, PMF has higher magnetic flux density $(117 \mathrm{mT})$ and Lorentz force $(0.103 \mathrm{~N})$ compared to HMF, which are $88 \mathrm{mT}$ and $0.087 \mathrm{~N}$, respectively. In addition, PMF is also found to have higher penetrability.

It also should be noted that under PMF, two vortices with opposite flow direction appear and lead to a pair of very strong
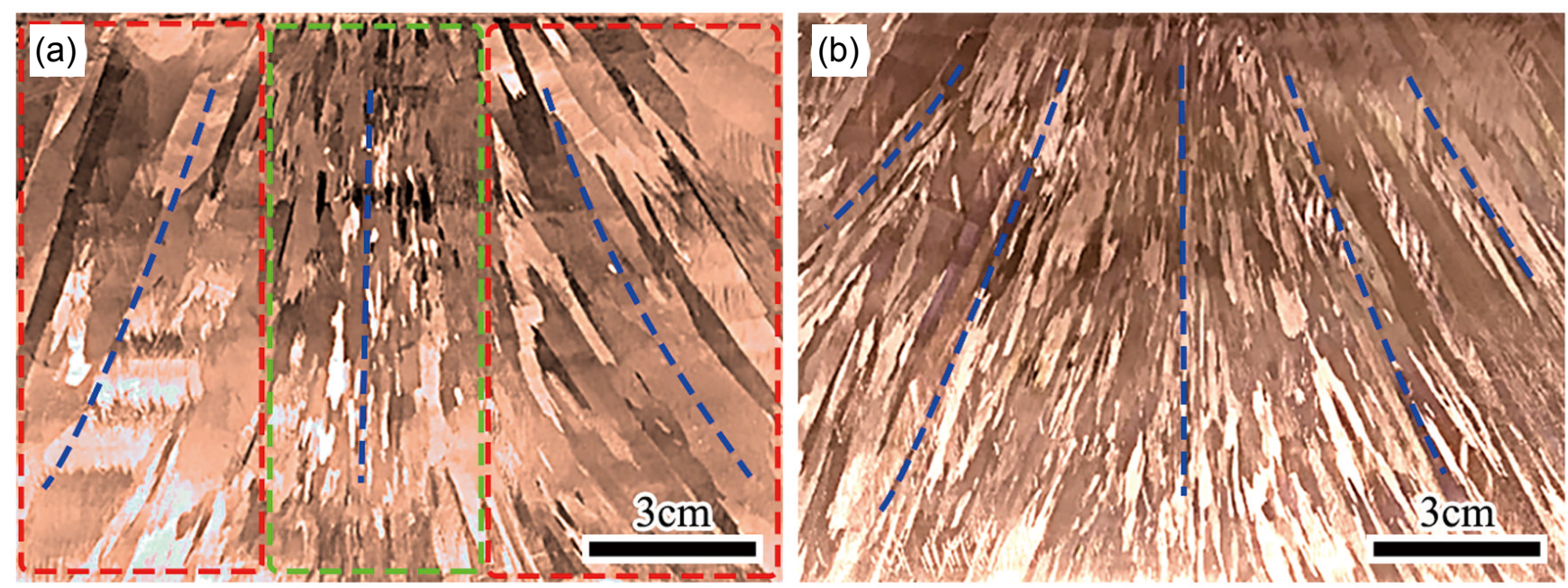

Fig. 8: Microstructure of billets by HMF (a) and PMF (b) casting 
dual convection flows, which enhances the impact of liquid flow on the edge of liquid pool and reduces the curvature radius of liquid pool. This mechanism causes the solidification front to be close to horizontal and reduces the angle between grain growth direction and casting direction.

\section{Conclusions}

(1) Maximum magnetic flux density and penetrability of PMF are significantly higher than those of HMF, because of the more rapid variation in transient current and higher peak value of magnetic flux density by PMF.

(2) PMF drives a higher Lorentz force and penetration depth than HMF does, because HMF creates a higher eddy current and reverse electromagnetic field which weakens the original electromagnetic field.

(3) PMF shows significant microstructure improvement due to the higher Lorentz force, uniform temperature field and strong dual convection.

\section{Acknowledgement}

This work was financially supported by the National Key Research and Development Program of China (Grant No. 2017YFB0305504).

\section{References}

[1] Chao Y B, Ling $\mathrm{C} \mathrm{H}$. Manufacture and application of sputtering target materials. Vacuum, 2001, 3: 11-15.

[2] Jiang L, Zhang L, Liu Z Q. Optimal design of Co/ln/Cu sputtering target assembly using finite element method and taguchi method. Acta Metallurgica Sinica, 2019, 32(11): 1407-1414.

[3] Verma B, Mishra S K. Spectral and structural investigation of layered growth of copper and graphene deposited by sputtering and annealing. Applied Physics A, 2019, 125(8): 534.1-534.11.

[4] Chen X R, Ning F K, Hou J, et al. Dual-frequency ultrasonic treatment on microstructure and mechanical properties of ZK60 magnesium alloy. Ultrasonics Sonochemistry, 2018, 40: 433441.

[5] Lichy P, Cagala M. Microstructure and thermomechanical properties of magnesium alloys castings. Archives of Foundry Engineering, 2012, 12(2): 49-54.
[6] Kabeel A E, El-Said E M S, Dafea S A, et al. A review of magnetic field effects on flow and heat transfer in liquids: present status and future potential for studies and applications. Renewable \& Sustainable Energy Reviews, 2015, 45: 830837.

[7] Li J T, Xu G M, Yu H L, et al. Improvement of AA5052 sheet properties by electromagnetic twin-roll casting. International Journal of Advanced Manufacturing Technology, 2016, 85(5-8): 1007-1017.

[8] Guo S J, Cui J Z, Le Q C, et al. The effect of alternating magnetic field on the process of semi-continuous casting for AZ91 billets. Materials Letters, 2005, 59(14-15): 1841-1844.

[9] Fu J, Yang Y. Microstructure and mechanical properties of Mg-Al-Zn alloy under a low-voltage pulsed magnetic field. Materials Letters, 2012, 67(1): 252-255.

[10] Kolesnichenko A F, Podoltsev A D, Kucheryavaya I N. Action of pulse magnetic field on molten metal. ISIJ International, 1994, 34: 715-721.

[11] Chen Q P, Shen H F. Numerical study on solidification characteristics under pulsed magnetic field. International Journal of Heat and Mass Transfer, 2018, 120: 997-1008.

[12] Haratmeh H E, Arezoodar A F, Farzin M. Numerical and experimental investigation of inward tube electromagnetic forming. International Journal of Advanced Manufacturing Technology, 2017, 88(5-8): $1175-1185$.

[13] Li H, Liu S C, Jie J C, et al. Effect of pulsed magnetic field on the grain refinement and mechanical properties of 6063 aluminum alloy by direct chill casting. International Journal of Advanced Manufacturing Technology, 2017, 93(9-12): 30333042.

[14] Li M J, Tamura T, Omura N, et al. The solidification behavior of the AZ61 magnesium alloy during electromagnetic vibration processing. Journal of Alloys and Compounds, 2010, 494(1-2): $116-122$.

[15] Natarajan T T, El-Kaddah N. Finite element analysis of electromagnetic and fluid flow phenomena in rotary electromagnetic stirring of steel. Applied Mathematical Modelling, 2004, 28(1): 47-61.

[16] Javurek M, Barna M, Gittler P, et al. Flow modelling in continuous casting of round bloom strands with electromagnetic stirring. Steel Research International, 2008, 79(8): 617-626.

[17] Bao L, Zhang Z Q, Le Q C, et al. Heat transfer behavior of AZ80-1\%Y alloy during low-frequency electromagnetic casting. Transactions of Nonferrous Metals Society of China, 2015, 25(11): 3618-3624.

[18] Jia $Y H$, Chen $X R$, Le $Q$ C, et al. Numerical study on action of HMF, PMF, DHMF, and DPMF on molten metal during electromagnetic casting. International Journal of Advanced Manufacturing Technology, 2019, 103(1-4): 201-221. 